\title{
Predicting Very Early Stage Mild Cognitive Impairment Based on a Voxel-wise Arterial Spin Labeling Analysis
}

\author{
Gloria Díaz ${ }^{1}$, Pablo García-Polo ${ }^{2}$, Virginia Mato ${ }^{3}$, Eva Alfayate ${ }^{4}$, \\ Juan Antonio Hernández-Tamames ${ }^{3}$, and Norberto Malpica ${ }^{3}$ \\ 1 Antonio Nariño University, Colombia \\ 2 Madrid-MIT M+Vision Consortium \\ 3 Rey Juan Carlos University, Spain \\ 4 Fundación CIEN, Spain
}

\begin{abstract}
In recent years, medical images have been increasingly used as an objective method for the diagnosis of neurodegenerative diseases. Most previous studies have been based on structural or functional magnetic resonance imaging. However, the results are not yet sufficient to identify early stages of dementia. In this paper, we present an image processing and pattern recognition strategy that allows to predict short-term conversion to Mild Cognitive Impairment (MCI) based on the analysis of Arterial Spin Labeling images. Healthy subjects, categorized as individuals at risk of dementia, were assessed annually in order to identify those that converted to MCI. After $1-2$ years, 20 subjects were classified as non-converters and 15 as converters according to the Mini-Mental State Examination test and other neuropsychiatric scales. The proposed approach was able to classify converter from non-converter subjects with an accuracy of 0.88 using the leave-one-out cross-validation method.
\end{abstract}

\section{Introduction}

Neurodegenerative diseases are characterized by a progressive loss of neurons in the central nervous system. It is estimated that more than 24 million elderly people are affected by these disorders, and this number is growing as the aging population increments. Mild cognitive impairment (MCI) refers to a brain function syndrome characterized by significant cognitive impairments that exceed those expected related to age [5]. This syndrome is considered as a transitory stage between normal cognitive function and Alzheimer's disease (AD). Consequently, early detection of MCI could be the key for timely therapeutic interventions that would delay the appearance of symptoms and preserve function and autonomy of the patients as long as possible.

Machine learning algorithms have been used to discriminate pathological from control subjects based on structural magnetic resonance images 9, Positron Emission Tomography (PET) or Single-Photon Emission Tomography (SPECT) functional volumes [15, fMRI brain activation maps [14 and Diffusion Tensor Images [8]. However, the prediction of early stages of the disease remains an unsolved problem due to the difficulty in detecting minimum brain changes.

E. Bayro-Corrochano and E. Hancock (Eds.): CIARP 2014, LNCS 8827, pp. 714721 2014.

(C) Springer International Publishing Switzerland 2014 
Arterial Spin Labeling (ASL) has become a popular magnetic resonance technique in brain research imaging, as it is the only non-invasive technique capable of measuring blood perfusion. It thus represents a great advantage over contrast agent based methods, such as $15 \mathrm{O}$ enriched H2O PET (Positron Emission Tomography) or Gadolinium-based DSC-MRI (Dynamic Susceptibility Contrast Magnetic Resonance Imaging). The basic principle of ASL is to employ the blood water itself as contrast agent to measure perfusion. For cerebral blood flow $(\mathrm{CBF})$ in the brain, this is attained by tagging a small bolus of arterial blood in the carotid arteries. The magnetization of inflowing blood water protons is inverted in the region of the carotid arteries were an external radiofrequency pulse is applied either as a short pulse $(10-20 \mathrm{~ms})$ or as a continuous or pseudocontinuous burst of RF $(1-2 s)$ in the presence of a gradient. After a period of time (post-labeling delay), blood is delivered to the entire brain through the smaller arteries and capillaries. This labeled arterial blood signal gives a rise to a reduction in the image intensity when compared to a non-labeled (control) image. The control and labeled images are subtracted and scaled to produce a final quantitative perfusion image. This image will reflect the amount of arterial blood delivered to each voxel within the slice within the inversion time; and through the use of a suitable model, can be expressed in conventional physiological units of $\mathrm{ml}$ - blood/100gtissue/min.

The availability of ASL as a routine method for assessment of basal CBF data has provided the possibility to examine brain physiology and generate a marker to probe functional differences between groups 1 . ASL is increasingly used in clinical studies of cerebral perfusion and has shown its validity in measuring perfusion changes in several neurodegenerative diseases including Alzheimer Disease (AD) [17/2]. Therefore, arterial spin labeling (ASL) data also begin to be used as biomarker for diagnosing probable Alzheimer's disease [10, even in an early stage [3]. However, to the best of our knowledge, there are no previous works on predicting the conversion of healthy patients to MCI onset. This paper evaluates the use of ASL data for classifying healthy subjects, identified as risk dementia, between short-term MCI converters and non-converters. The proposed approach extracts statistical features that describe volume regions identified as those which exhibit hypoperfusion patterns in a voxel-wise analysis. These features are used for training a machine learning approach, which is able to separate subjects that will convert to MCI from those that will continue healthy at least during the next two years.

\section{Materials and Methods}

Figure 1 illustrates the main stages of the proposed approach. In an off-line process, ASL-MRI images of converters and non-converters subjects are processed in order to automatically extract regions which exhibit hypoperfusion in a statistical voxel-wise between groups analysis. A feature extraction process is then applied to generate descriptors of those regions, which are used for training a learning model able to separate the feature space into the two classes. Once the 
classifier is designed, when a new subject's ASL-MRI is presented, the selected regions are located and characterized with the same descriptors used in the training stage, and the trained model is used to classify the individual subject into converter or non-converter.

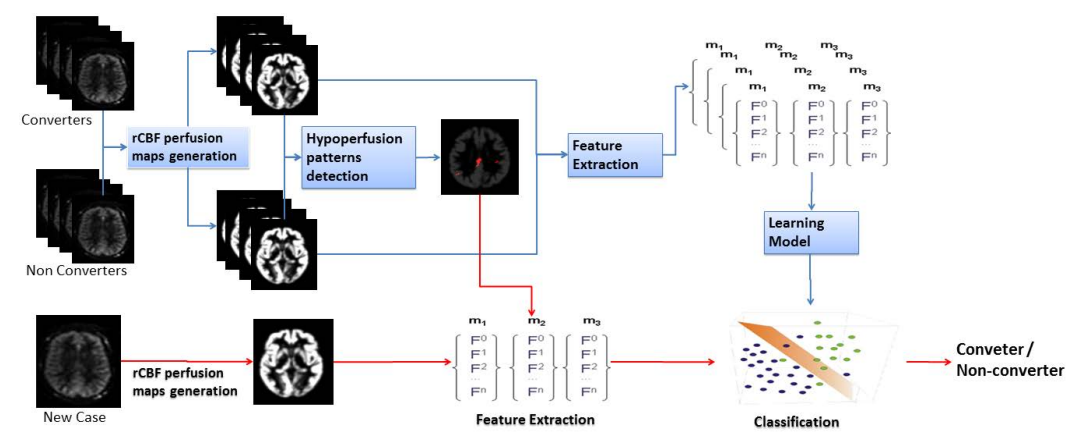

Fig. 1. Proposed approach. Blue lines illustrate the learning model workflow. Red lines illustrate the classification of a new subject.

\subsection{Image Acquisition and Preprocessing}

Participants underwent MRI examination in a 3T Signa HDx MR scanner (GE Healthcare, Waukesha, WI) using an eight-channel phased array coil. The first sequence was a $3 D-T 1$ weighted SPGR with a $T R=10.024 \mathrm{~ms}$, TE $=4.56 \mathrm{~ms}$, $T I=600 \mathrm{~ms}, N E X=1$, acquisition matrix $=288 \times 288$, full brain coverage, resolution $=1 \times 1 \times 1 \mathrm{~mm}$, flip angle $=12$. The second sequence was a $3 \mathrm{D}$ pCASL pulse sequence with full brain coverage, matrixsize $=128 \times 128$, resolution $=$ $1.875 x 1.875 x 4 \mathrm{~mm}$, flipangle $=155$, labelingtime $=1.5 \mathrm{~s}$, post-labelingdelay $=$ $2.025 \mathrm{~s}, T R=4.733 \mathrm{~s}, \mathrm{TE}=9.812 \mathrm{~ms}, \mathrm{NEX}=3$, acquisitiontime $=6 \mathrm{~min}$ that was used to generate the regional cerebral blood flow (rCBF) maps.

The 3DT1 weighted images were first segmented with the FreeSurfer image analysis suite into cortical and subcortical structures for volume assessment, as described in previous works [16]. Briefly, this processing includes motion correction of volumetric T1 weighted images, removal of non-brain tissue using a hybrid watershed/surface deformation procedure, automated Tailarach transformation, segmentation of the subcortical white matter and deep gray matter volumetric structures (including hippocampus, amygdala, caudate, putamen, ventricles) intensity normalization and parcellation of the cerebral cortex based on the gyral and sulcal structures. Then, a SPM8 and DARTEL tool analysis was performed over the anatomical images to obtain the gray matter (GM), white matter (M) and cerebro spinal fluid (CSF) segmentations as well as their respective volumes in litres [12. The DARTEL tool from SPM8 provides high dimensional warping deformation maps, which transform each subject space to the Montreal Neurological Institute (MNI) space. This deformation maps can be obtained for the GM, WM or CSF segmented images. 
It is known that GM perfusion is 3 times greater than WM perfusion [11. Thus rCBF images closely resemble a GM probabilistic map. Finally, an inter-modality co-registration between the ASL perfusion maps and the GM images allows the application of the high dimensional warping deformation maps obtained with the DARTEL tool, therefore obtaining MNI normalized and smoothed $(F W H M=$ $4 x 4 x 4 \mathrm{~mm}) \mathrm{rCBF}$ perfusion maps for each subject.

\section{2 rCBF Based Feature Extraction}

In the pre-processing stage all images are aligned, i.e. a voxel in any image approximately corresponds to the same voxel in another image, so that an $\mathrm{CBF}$ comparison at every voxel between groups can be carried out. The general linear model (GLM) was used to identify regions in which CFB exhibit significant hypoperfusion patterns in the training data. A hypothesis two-sample t-test was used to compute a statistical map that was thresholded for selecting voxels which meet these two conditions: their value was statistically significantly $(p<0.001)$ and they could be grouped together within regions with more than 50 voxels with similar statistical properties. Age, genre, GM volume and left hippocampal volume were used as covariates.

Therefore, a set of regions scattered through the rCBF maps, were identified as regions of interest, and then each of them was modeled as a random variable, described by its corresponding probability density function, and their first three statistical order moments were computed and used as region descriptor. The final feature vector used for describing each subject resulted from the concatenation of the statistical moments of all detected regions.

\subsection{SVM Classification}

Based on the regional features selected in the previous stage, a support vector machine (SVM) learning model was trained for classifying individual ASL-MRI images, which has been shown to be a powerful tool for statistical pattern recognition in neuroimaging-based clinical prediction [815. SVM produces an optimal separating hyperplane between two classes in a feature space, in which each training instance has been represented. These hyperplane corresponds to the largest separation, or margin, between the two classes. When a new instance should be classified, original feature vector is mapped to the same space and the distance to SVM hyperplane allows to decide if the new instance falls into one category or the other. In general, the original SVM algorithm proposed by Vladimir Vapnik 13. was a linear discriminator, but subsequent modifications suggested that nonlinear discrimination tasks can be achieved thanks to the "kernel trick". This is, mapping the original non-linear observations into a higher-dimensional space in which boundaries among classes become linear. In this work, the Gaussian radial basis function and polynomial kernels were evaluated [13. 


\section{$3 \quad$ Experimental Results}

\subsection{Participants}

Participants were selected from the "Proyecto Vallecas" trial, which has been designed to assess normal healthy ageing and the appearance of neurodegenerative diseases, in particular Alzheimer disease. All subjects were in the age between 70 and 85 years and were first included in the study as healthy subjects based on several psychological and neurological tests, including the Mini-Mental State Examination (MMSE) [6], the Grober and Buschke test [7] and the Geriatric Depression Scale [18. All subjects underwent MRI examination as well as psychological and neurological assessment every 6-12 months. Informed consent was obtained from all participants prior to evaluation.

One of the support features in the diagnostic criteria for $\mathrm{AD}$ is the presence of medial temporal lobe atrophy [4, that leads to volume loss in the hippocampi, the entorhinal cortex and the amygdala, evidenced on MRI. The subjects included in the sample were classified according to this feature. For doing so, the normalized value Left and Right Hippocampi Volume (LH, RH) was computed from the segmented 3DT1 images and then divided by the total grey matter volume. A total of 35 subjects with left and right hippocampi volume below the media were identified at risk of dementia and were included in this study. Additionally, some of the started to show some cognitive deterioration (after the second or third examination) and were classified by the physicians as mild cognitive impairment (MCI) subjects, according the psychological and neurological tests described above. This process leads to a split of this group of 35 subjects risk of developing $\mathrm{AD}(\mathrm{RAD})$ with medial temporal lobe atrophy, in a group of 15 converters to $\mathrm{MCI}$ subjects and another group of 20 non-converters subjects.

\subsection{Detection of Perfusion Changes Patterns}

Figure 2 shows variations in perfusion between converters and non-converters subjects using the two-sample t-test presented above $(p<0.001)$. Changes in perfusion are depicted in the left and right temporal lobes, left and right insula, medial cingulate gyrus and anterior cingulate, cuneus and precuneus.

\subsection{Classification Performance}

SVM learning models were trained through an exhaustive search of their learning parameters. The regularization parameter $C$ was varied from 1 to 10 with increment steps of 1 , while the parameters $E$ and $\gamma$ that defines the nonlinear mapping from input space to some high-dimensional feature space, were varied from 1 and 5 and from 0.1 and 1 with increment steps of 1 and 0.2 , respectively. The effect of using uniquely the first order moment and the combination of the first two and first three order moments as region descriptors was also evaluated.

Because the number of instances of each class is small, we used the well-known leave-one-out cross validation strategy for evaluating the proposed approach. 


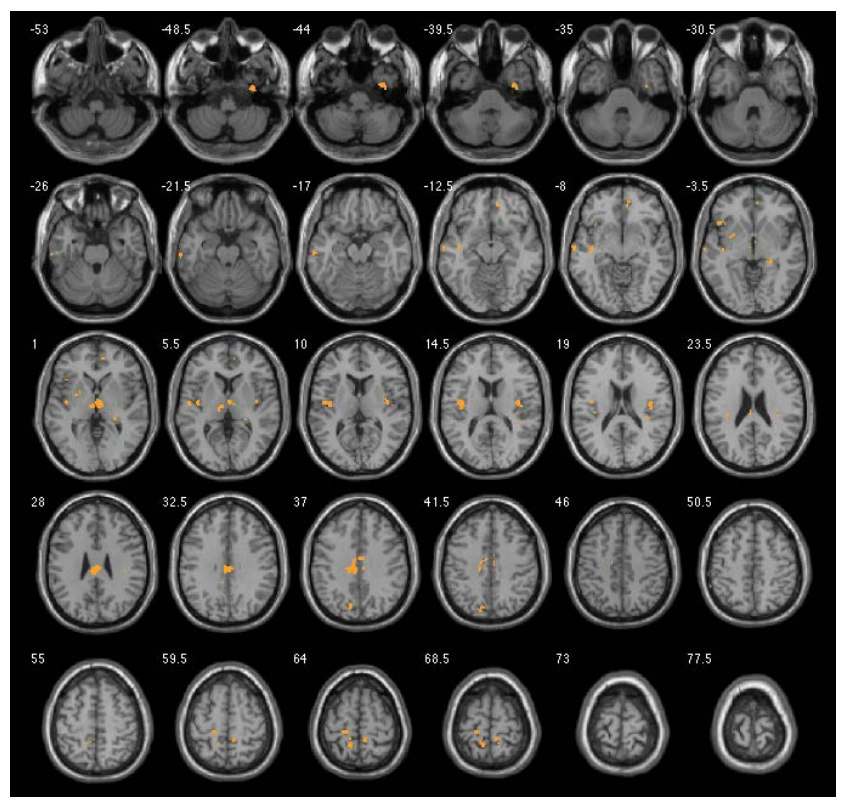

Fig. 2. Risk subjects Vs. Converters in ASL

For doing so, each test consisted on leave a subject out and executing all the training process with the remaining 34 , i.e. at each test the 34 subjects are used to perform the statistical test that allows to extracting the perfusion variation regions and then the location of these regions is used to extract the $\mathrm{rCBF}$ values that describe both training and test subjects. Classification performance was quantified in terms of its predictive accuracy, sensitivity, specificity and area under the ROC curve.

From a comprehensive analysis of all parameter combination, we found that the number of moments used is the parameter that has the largest effect on general performance, whilst Complexity $(C)$, exponent $E$ and $\gamma$ of the SVM learning model did not have relevant effect (results not shown). Therefore, the best values of these parameters are used to obtain the results reported here, i.e. $C=2.0, \gamma=0.1$ and $E=3.0$. Classification results are reported in the Table 1. Results show that the proposed approach achieves the best performance using the polynomial kernel and 3 statistical moments. The reason for this is that the feature descriptors are extracted from regions that share statistical properties, but contain different perfusion values whose distribution is captured by the proposed approach. 
Table 1. Classification performance for Polynomial and RBF kernels using 1, 2 and 3 statistical moments as region descriptors

\begin{tabular}{|c|c|c|c|c|c|c|c|c|}
\hline & \multicolumn{4}{|c|}{ Polynomial Kernel } & \multicolumn{4}{|c|}{ RBF Kernel } \\
\hline Moments & Acc. & Sens. & Spec. & $A U C$ & Acc. & Sens. & Spec. & $A U C$ \\
\hline 1 & 0.68 & 0.73 & 0.65 & 0.68 & 0.74 & 0.80 & 0.70 & 0.74 \\
\hline 2 & 0.82 & 0.66 & 0.95 & 0.85 & 0.71 & 0.73 & 0.70 & 0.71 \\
\hline 3 & 0.88 & 0.80 & 0.95 & 0.89 & 0.85 & 0.86 & 0.85 & 0.85 \\
\hline
\end{tabular}

\section{Conclusions}

In this paper a pattern classification approach for classifying subjects according to their brain perfusion data was presented. This approach looks for relevant regions in ASL-MRI images, which are identified as discriminative to the classification task. The approach has been tested on a study on healthy subjects that converted to MCI then of two years, providing promising results on the prediction of very early stages of dementia.

In this study we demonstrate that standard statistical tests that are frequently applied in groups comparisons can be used for automatically extracting regions that contain relevant information for diagnostic purposes.

For the future, we plan to tackle the multiclass classification problem, which requires that more sophisticated feature extraction process and machine learning approaches be investigated. Moreover, we plan to integrate or combine multimodal information such as ASL, MRI, DTI and neuropsychological test results in order to look for other patterns that provide most discrimination power for assisting in the early diagnosis of neurodegenerative diseases.

\section{References}

1. Austin, B.P., Nair, V.A., Meier, T.B., Xu, G., Rowley, H.A., Carlsson, C.M., Johnson, S.C., Prabhakaran, V.: Effects of hypoperfusion in Alzheimer's disease. Journal of Alzheimer's Disease: JAD 26(suppl. 3), 123-133 (2011)

2. Binnewijzend, M.A.A., Kuijer, J.P.A., Benedictus, M.R., van der Flier, W.M., Wink, A.M., Wattjes, M.P., van Berckel, B.N.M., Scheltens, P., Barkhof, F.: Cerebral blood flow measured with 3D pseudocontinuous arterial spin-labeling MR imaging in Alzheimer disease and mild cognitive impairment: a marker for disease severity. Radiology 267(1), 221-230 (2013)

3. Bron, E.E., Steketee, R.M.E., Houston, G.C., Oliver, R.A., Achterberg, H.C., Loog, M., van Swieten, J.C., Hammers, A., Niessen, W.J., Smits, M., Klein, S.: Diagnostic classification of arterial spin labeling and structural MRI in presenile early stage dementia. Human Brain Mapping (April 2014)

4. Dubois, B., Feldman, H.H., Jacova, C., Dekosky, S.T., Barberger-Gateau, P., Cummings, J., Delacourte, A., Galasko, D., Gauthier, S., Jicha, G., Meguro, K., O’brien, J., Pasquier, F., Robert, P., Rossor, M., Salloway, S., Stern, Y., Visser, P.J., Scheltens, P.: Research criteria for the diagnosis of alzheimer's disease: revising the nincds-adrda criteria. Lancet Neurology 6(8), 734-746 (2007), lR: 20101118; JID: 101139309; 0 (Amyloid beta-Peptides); 0 (tau Proteins); CIN: Int. Psychogeriatr. 20(4), 853-855 (2008), PMID: 18416874; CIN: Lancet Neurol. 6(8), 667-669 (2007), PMID: 17616483; CIN: Lancet Neurol. 7(8), 668-670 (2008), PMID: 18635012; RF: 143; Publish 
5. Ewers, M., Sperling, R.A., Klunk, W.E., Weiner, M.W., Hampel, H.: Neuroimaging markers for the prediction and early diagnosis of Alzheimer's disease dementia. Trends in Neurosciences 34(8), 430-442 (2011)

6. Folstein, M.F., Folstein, S.E., McHugh, P.R.: "Mini-mental state". a practical method for grading the cognitive state of patients for the clinician. Journal of Psychiatric Research 12(3), 189-198 (1975)

7. Grober, E., Buschke, H.: Genuine memory deficits in dementia. Developmental Neuropsychology 3(1), 13-36 (1987)

8. Haller, S., Missonnier, P., Herrmann, F.R., Rodriguez, C., Deiber, M.P., Nguyen, D., Gold, G., Lovblad, K.O., Giannakopoulos, P.: Individual classification of mild cognitive impairment subtypes by support vector machine analysis of white matter DTI. AJNR. American Journal of Neuroradiology 34(2), 283-291 (2013)

9. Klppel, S., Abdulkadir, A., Jack Jr., C.R., Koutsouleris, N., Mouro-Miranda, J., Vemuri, P.: Diagnostic neuroimaging across diseases. NeuroImage 61, 457-463 (2012)

10. Mak, H.K.F., Qian, W., Ng, K.S., Chan, Q., Song, Y.Q., Chu, L.W., Yau, K.K.W.: Combination of MRI Hippocampal Volumetry and Arterial Spin Labeling MR Perfusion at 3-Tesla Improves the Efficacy in Discriminating Alzheimer's Disease from Cognitively Normal Elderly Adults. Journal of Alzheimer's Disease: JAD (March 2014)

11. Parkes, L.M., Rashid, W., Chard, D.T., Tofts, P.S.: Normal cerebral perfusion measurements using arterial spin labeling: reproducibility, stability, and age and gender effects. Magnetic Resonance in Medicine: Official Journal of the Society of Magnetic Resonance in Medicine / Society of Magnetic Resonance in Medicine 51(4), 736-743 (2004), http://www.ncbi.nlm.nih.gov/pubmed/15065246

12. Pernet, C., Andersson, J., Paulesu, E., Demonet, J.F.: When all hypotheses are right: a multifocal account of dyslexia. Human Brain Mapping 30(7), 2278-2292 (2009), http://www.ncbi.nlm.nih.gov/pubmed/19235876

13. Platt, J.C.: Fast training of support vector machines using sequential minimal optimization, pp. 185-208 (February 1999)

14. Preti, M.G., Makris, N., Papadimitriou, G., Laganà, M.M., Griffanti, L., Clerici, M., Nemni, R., Westin, C.F., Baselli, G., Baglio, F.: A novel approach of groupwise fMRI-guided tractography allowing to characterize the clinical evolution of Alzheimer's disease. PloS One 9(3), e92026 (2014)

15. Ramírez, J., Górriz, J., Salas-Gonzalez, D., Romero, A., López, M., Álvarez, I., Gómez-Río, M.: Computer-aided diagnosis of Alzheimer's type dementia combining support vector machines and discriminant set of features. Information Sciences 237, 59-72 (2013)

16. Reuter, M., Schmansky, N.J., Rosas, H.D., Fischl, B.: Within-subject template estimation for unbiased longitudinal image analysis. NeuroImage 61(4), 1402-1418 (2012)

17. Wang, Z., Das, S.R., Xie, S.X., Arnold, S.E., Detre, J.A., Wolk, D.A.: Arterial spin labeled mri in prodromal alzheimer's disease: A multi-site study. NeuroImage Clinical 2, 630-636 (2013)

18. Yesavage, J.A., Brink, T.L., Rose, T.L., Lum, O., Huang, V., Adey, M., Leirer, V.O.: Development and validation of a geriatric depression screening scale: A preliminary report. Journal of Psychiatric Research 17(1), 37-49 (1982-1983) 